\title{
« Macht keine Geschichten »
}

Matière, fiction, histoire : du performatif dans la prose de Wolfgang Hilbig?

"Macht keine Geschichten“. Materie, Fiktion, Geschichte: Performativität in Wolfgang Hilbigs Prosa?

"Macht keine Geschichten". Material, Fiction, History: Performativity in Wolfgang Hilbig's Prose?

\section{Bénédicte TERRISSE}

\section{(2) OpenEdition}

\section{Journals}

Édition électronique

URL : http://journals.openedition.org/ceg/3531

DOI : $10.4000 /$ ceg.3531

ISSN : 2605-8359

\section{Éditeur}

Presses Universitaires de Provence

Édition imprimée

Date de publication : 15 novembre 2018

Pagination : $79-94$

ISBN : 979-10-320-0183-7

ISSN : 0751-4239

\section{Référence électronique}

Bénédicte TERRISSE, « « Macht keine Geschichten » », Cahiers d'Études Germaniques [En ligne], 75|

2018, mis en ligne le 25 avril 2020, consulté le 29 novembre 2020. URL : http://

journals.openedition.org/ceg/3531 ; DOI : https://doi.org/10.4000/ceg.3531 


\section{"Macht keine Geschichten " \\ Matière, fiction, histoire : du performatif dans la prose
de Wolfgang Hilbig?}

Bénédicte TERRISSE

Université de Nantes/ CRINI

" Comment la littérature entre-t-elle dans la vie? Quel est son point d'impact? son degré d'activité » écrit Marielle Macé en introduction à l'article de Jonathan Culler intitulé "Philosophie et littérature : les fortunes du performatif ${ }^{1}$."

Cette question peut se lire comme l'un des enjeux possibles du recours à la matérialité dans la prose de l'écrivain. La matérialité jouerait le rôle d'، interface entre la littérature et le réel $^{2}$ ". Elle permettrait de transformer l'énonciation littéraire en acte qui accomplit ce à quoi elle fait référence. Si le langage littéraire lui-même est événement, et pas seulement description d'un événement, c'est, d'une part, parce qu'il comporte une dimension autoréflexive qui le met en scène lui-même et, d'autre part, parce qu'il parvient à " faire exister les personnages et les événements dont [l'œuvre] raconte l'histoire ${ }^{3}$ ". Mettre en scène la matérialité serait l'un des moyens utilisés par Hilbig "pour faire exister [les] choses qu'[il] nomme ${ }^{4}$ ". La présente contribution s'attache à débusquer les dispositifs narratifs qui permettent la mise en scène de la matérialité et confèrent par-là une dimension performative aux textes.

La matière prend une résonance particulière au regard de la trajectoire biographique de l'écrivain. Après une formation de tourneur-fraiseur, Hilbig (1941-2007) travailla pendant vingt ans dans différents secteurs de l'industrie. Il fut notamment ouvrier-chauffeur dans les chaufferies de fonderies et blanchisseries est-allemandes avant d'obtenir en 1980 le statut officiel d'écrivain en RDA. La matière, c'est ici, très concrètement, le feu, la cendre et le charbon, mais aussi les exsudations corporelles de celui qui se tient au contact de ces éléments. L'un des enjeux des textes de Hilbig est de tenter de penser ensemble ces deux identités, d'arpenter en écriture les points de contact entre l'activité

1. Marielle Macé, "Note introductive à Jonathan Culler, "Philosophie et littérature : les fortunes du performatif" ", traduit par Marie de Gandt, Littérature 144, 2006/ 4, p. 81-100, ici p. 81. DOI 10.3917/litt.144.0081.

2. Ibid.

3. Ibid., p. 90.

4. Ibid., p. 87. 
d'écrivain et celle d'ouvrier, de convertir la littérature dans la vie, la vie dans la littérature. La matière constitue l'un de ces points de passage.

Enfin, la question de la matière occupe le premier plan d'un corpus de textes qui ont en commun leur appartenance au genre des récits ${ }^{5}$. Hilbig est l'auteur de trois romans et de trois recueils de poésie ${ }^{6}$. Cependant, la plus grande partie de sa production a pris la forme de textes portant l'étiquette vague de "Prosa ", "Kurzprosa ", "Prosastücke " ou "Erzählungen" ". Rassemblés pour la plupart en recueil, ils se définissent par leur non-appartenance au roman et à la poésie. Y aurait-il un rapport entre la prédilection pour la question de la matérialité et cette forme à l'identité générique un peu floue ?

Nous croiserons les deux questions que sont, d'une part, le rapport entre matérialité et performatif ainsi que les techniques narratives utilisées, et, d'autre part, les conséquences génériques de la mise en scène de la matière, et procéderons en trois temps. Nous nous pencherons en premier lieu sur les objets, en particulier les textes-objets, en analysant comment, dans le recueil Der Brief (1985), les procédés narratifs qui mettent en scène la transformation du livre en objet circulant renforcent l'appartenance des textes au genre du récit fictionnel. Ce faisant, nous mettrons en lumière le mode de lecture que ces textes-objets impliquent. Dans un deuxième temps, nous étudierons la manière dont matière et récit s'articulent dans Die Kunde von den Bäumen (1994) en montrant notamment comment s'y élabore, sur le modèle de la lettre de Lord Chandos ${ }^{8}$, une poétique de la matière inscrite dans une crise du récit. Après ces exemples articulant de manière contradictoire matérialité et narrativité, nous reviendrons sur les procédés décrits dans les deux parties comme pouvant relever du performatif, c'est-à-dire mettant en scène un passage des frontières entre la littérature et la vie, afin de montrer qu'ils ont pour fonction de répondre au problème de la référence.

5. Cf. Gillian Pye, "Trash and Transformation : The Search for Identity in Wolfgang Hilbig's Die Kunde von den Bäumen and Alte Abdeckerei ", New German Critique 39, 2012, n 2/ 116, p. 87-102.

6. Les romans sont Eine Übertragung (1989), "Ich " (1993) et Das Provisorium (2000). Les recueils de poésie sont abwesenheit (1979), die versprengung (1986) et Bilder vom Erzählen (2001), tous parus aux éditions Fischer.

7. On retrouve ces sous-titres génériques pour les volumes des Hilbig-Werke, publiés chez Fischer, ou bien le recueil Über den Tonfall. Drei Prosastücke, Berlin, Friedenauer Presse, 1990. Le sous-titre du seul recueil paru en RDA, Stimme Stimme, Leipzig, Reclam, 1983, est parlant lui aussi : "Gedichte und Prosa ". Le pendant logique de " Gedichte " aurait dû être " Erzählungen ". "Prosa " signale l'indétermination générique des textes non écrits en vers de Hilbig.

8. Hugo von Hofmannsthal, "Ein Brief ", in Hofmannsthal, Erzählungen, erfundene Gespräche und Briefe, Reisen, Frankfurt a. M., Fischer Taschenbuch Verlag, 1979 [1902], p. 461-472. 


\section{Textes en circulation et fiction}

\section{L'objet lettre et la fiction en question}

C'est d'abord à travers les objets, et en particulier le texte comme objet, que se manifeste la matérialité dans l'œuvre de Wolfgang Hilbig. Très tôt, le texte n'est pas le simple support abstrait, quasi-immatériel, d'un contenu, d'une histoire ou bien d'idées. Au contraire, il joue un rôle dans les récits, entre dans la fiction, où il apparaît comme quelque chose que l'on peut appréhender par le sens du toucher, tenir entre ses mains : il est un objet qui circule et qui, par là-même, peut nous échapper. Michael Niehaus fait de la capacité à circuler l'un des traits définitoires de l'objet' .

Emblématique à la fois de la mise en scène du texte en objet circulant et du mode de lecture autoréflexif qu'elle implique, la lettre est, avec le motif du vol de manuscrit, un topos de la prose hilbigienne depuis les années 1980. La lettre incarne par excellence le texte dans sa dimension matérielle d'objet. Circulant entre un expéditeur et un destinataire, le morceau de papier recouvert d'une enveloppe est, en RDA, pris en charge par une institution étatique ${ }^{10}$ pour délivrer son message ou son contenu. La lettre semble ainsi pouvoir représenter en elle-même le fonctionnement du langage à la fois comme moyen de communication qui passe par le contrôle d'une autorité et comme structure reposant sur l'attelage d'une variante du signifiant (le papier ou la lettre de l'alphabet) et d'un équivalent du signifié (le message) ${ }^{11}$. Le motif de la lettre ajoute à la multitude des couches de lectures possibles du texte hilbigien des dimensions pragmatique et performative.

Le récit "Der Heizer ", écrit en 1980, et paru deux ans plus tard dans le recueil Unterm Neomond. Erzählungen en contient une première esquisse sous la forme d'une lettre de démission que le personnage ouvrier-chauffeur n'a jamais écrite mais qui pourrait bien avoir pris la forme du récit que nous lisons. D'emblée, lettre et récit de l'auteur se confondent. La superposition de la lettre et du texte que nous lisons investit de fait le récit d'une dimension performative : paru l'année où son auteur obtient l'immatriculation fiscale signalant que le régime le reconnaît officiellement comme écrivain, "Der Heizer " est à la fois un texte littéraire et un acte de démission de la condition ouvrière.

La lettre confère son titre au deuxième recueil en prose de l'écrivain, Der Brief. Drei Erzählungen, paru en $1985^{12}$. De nouveau, on est incité à lire le recueil et les récits comme constituant la véritable lettre. Le contenant (le texte)

9. Michael Niehaus, Das Buch der wandernden Dinge. Vom Ring des Polycrates bis zum entwendeten Brief, München, Carl Hanser Verlag, 2009.

10. Cf. Bernhard Siegert, Relais : Geschicke der Literatur als Epoche der Post, 1751-1913, Berlin, Verlag Brinkmann \& Bose, 1993.

11. Cf. Jacques Lacan, "Le séminaire sur "La lettre volée" ", in Lacan, Écrits I, Paris, Seuil, 1999 [1966], p. 11- 61.

12. Wolfgang Hilbig, "Der Brief ", in Hilbig, Der Brief. Drei Erzählungen, Frankfurt a. M., Fischer, 1985. 
et le contenu (ce qui est raconté) sont une nouvelle fois confondus. Dans ce dernier recueil, le motif du manuscrit envoyé par la poste où il y disparaît ("Der Brief ", $139{ }^{13}$; "Die Angst vor Beethoven ") transpose de manière littéraire un fait historique et biographique, celui de la surveillance du courrier par les agents de la Stasi qui intercepte les manuscrits, notamment ceux à destination des maisons d'édition ouest-allemandes ${ }^{14}$. C'est sans doute cette communication à double détente, entre expéditeur et destinataire, mais aussi entre l'expéditeur et une institution postale devenue haut lieu de la surveillance et du pouvoir, que mettent au jour les personnages de Hilbig lorsqu'ils se rendent au guichet pour s'envoyer une lettre à eux-mêmes ("Der Brief ", 142) ou bien écrivent leur lettre directement à une haute administration (" Er, nicht ich "15 ", 176). Ces textes relèvent de la fiction, par opposition au récit factuel, tout en revêtant une fonction pragmatique : ce sont autant de messages envoyés au pouvoir, qui, certes, a reconnu à leur auteur le statut d'écrivain mais n'a pas autorisé leur publication à l'Ouest. Retenant le moment de l'avant-publication, ils racontent l'incertitude de leur devenir objet-livre, la possibilité qu'ils gardent à jamais leur statut de manuscrit, disparaissent dans les rouages de la poste ou finissent enfouis dans les archives de la sécurité d’État.

Ainsi, l'intrigue du récit " $E r$, nicht ich " se tient tout entière dans l'acte, sans arrêt ajourné, d'aller porter une lettre à la boîte. L'ajournement est dû au contenu scandaleux de la lettre qui décrit confusément un meurtre, comme le révèlent les dernières pages du récit. Tout au long du texte, le personnage tente de se rappeler le contenu de la lettre et les circonstances de son écriture. On le voit, texte et lettre ne se recoupent pas complètement. Les textes orchestrent un jeu narratologique alternant distinction et confusion entre ce qui relève du récit et ce qui relève de la lettre. Le récit cite la lettre. La différence de niveau narratif est marquée de manière typographique par des italiques ("Er, nicht ich ", 186-187) ou des guillemets ("Der Brief ", 166-167). Ces conventions typographiques signalent davantage que le simple passage entre le niveau extradiégétique $d u$ récit-cadre et celui, intradiégétique, du récit interne, c'est-à-dire de la lettre. Elles indiquent un changement matériel concernant le support du récit : le récit passe d'une absence de support matériel, c'est-à-dire un récit raconté par la voix du narrateur, à un récit présenté comme écrit sur du papier. Cependant ces conventions n'ont qu'un pouvoir de suggestion, elles ne donnent pas à voir la lettre en tant que telle et peuvent être manipulées.

C'est sur cette incertitude que repose le dispositif métaleptique qui préside à la construction narrative de la plupart des récits contenant des lettres. En effet,

13. Dans cet article, le nombre placé entre parenthèses indique la page mentionnée.

14. "Scherben für damals und jetzt ", un recueil de poésie composé par Hilbig dans la première moitié des années 1960 a été retrouvé dans les archives de la Stasi au moment de la chute du Mur. Jürgen Hosemann, " Nachbemerkung zu dieser Ausgabe ", in Wolfgang Hilbig Werke. Gedichte, vol. 1, Frankfurt a. M., Fischer, 2008, p. 527. Les contacts avec Thomas Beckermann, lecteur chez Fischer, sont sans doute à l'origine de l'arrestation de Hilbig en 1978.

15. Wolfgang Hilbig, "Er, nicht ich ", in Hilbig, zwischen den paradiesen. Prosa Lyrik, Leipzig, Reclam, 1992, p. 134-189. 
la lettre, en principe enchâssée dans le récit, et le récit-cadre finissent par échanger leur position au point que l'on ne sait finalement plus si ce que l'on prenait pour la lettre n'est pas en réalité le récit et inversement : le " niveau où l'on raconte " et celui " que l'on raconte ${ }^{16}$ " finissent par se confondre. Après avoir cité quelques extraits de ses propres textes, le narrateur-écrivain $d u$ récit-cadre de "Der Brief" raconte l'histoire d'un personnage qui lui ressemble : celui-ci a des velléités d'écrivain et a, semble-t-il, assassiné une factrice, comme il l'avoue indirectement dans une lettre signée de sa main que le narrateur $d u$ récit-cadre cite pour preuve. Parce que ce dernier porte un stigmate à la main droite, il semble faire endosser à son personnage du récit intérieur le crime qu'il a en réalité commis lui-même. Les écrits produits par son personnage sont en fait de sa plume. C'est finalement la fragilité de la matière des récits et des lettres, de leur valeur d'attestation ou de preuve, de leur " réalité ", comparée au caractère tangible d'une blessure physique, que "Der Brief " met au jour.

On peut lire "Der Brief " comme un texte sur l'entorse ou la transgression : il franchit les frontières de la représentation (les niveaux extra- et intradiégétiques) comme son personnage franchit les barrières sociales séparant l'ouvrier de l'écrivain. Il commet une " entorse au pacte de la représentation ${ }^{17}$ " quand son narrateur s'est blessé à la main et raconte un crime en " transgressant le[s] seuil[s] d'enchâssement ${ }^{18}$ ". En un sens, la métalepse achève de classer les narrations étranges de Hilbig du côté de la fiction, puisque c'est un " moyen réservé au récit de fiction ${ }^{19}$ ".

Les conséquences de ces manipulations frauduleuses sur la fiction restent néanmoins ambiguës. Elles peuvent d'une part aiguiser la conscience $d u$ caractère conventionnel, voire arbitraire, de ces frontières entre les niveaux de la représentation et abolir ainsi toute illusion référentielle, toute croyance en la fiction. Inversement, la métalepse peut aussi paradoxalement, potentialiser l'illusion, intensifier l'immersion : donner le sentiment angoissant que la fiction se poursuit dans le monde du lecteur, dans une sorte de perpétuation presque infinie des passages de frontière ${ }^{20}$ qui renforce le pouvoir de la fiction.

\section{Papier et poésie}

Dans le texte-objet, des procédés comme la métalepse, mettent à l'honneur ce qui est typique des récits de fiction, tel le jeu avec les niveaux narratifs. Dans le texte-matière, au contraire, on peut se demander si la concentration sur la matière ne fonctionne pas comme une entrave au récit.

16. Gérard Genette, Discours du récit, Paris, Le Seuil, 2007 [1983], p. 245.

17. John Pier, Jean-Marie Scheffer (dir.), Métalepses. Entorses au pacte de la représentation, Paris, École des Hautes Études en Sciences Sociales, 2005.

18. Gérard Genette, Métalepse. De la figure à la fiction, Paris, Seuil, 2004, p. 14.

19. John Pier, Jean-Marie Schaeffer, "Introduction. La métalepse, aujourd'hui ", in Pier, Schaeffer (dir.), Métalepses, p. 7-15, ici p. 14.

20. Dorrit Cohn, " Métalepse et mise en abyme », in Pier, Schaeffer (dir), ibid., p. 121-130. 
Si la lettre que l'on cite ouvre un niveau narratif dans le texte, la lettre que l'on décrit dans sa substance comme une feuille de papier participe à l'élaboration de la signification globale du texte, en faisant abstraction de la dimension proprement narrative.

Ainsi, dans "Der Brief ", des feuilles d'arbres s'envolent et finissent par se mêler au papier entassé dans une entreprise de recyclage ("Altpapier "). Elles rappellent au narrateur sa fameuse lettre (132), au point qu'il se demande si le papier qui vole dans le ciel ne provient pas finalement du bâtiment de la poste qui jouxte la recyclerie (" Altstoffhandlung ", 133). La description obéit à une logique de pensée et d'observation associative, motivée par la ressemblance des choses entre elles et leur contiguïté. Elle semble cependant se doubler d'une rêverie sur les mots, matière du texte : les feuilles des arbres ("Blätter ", 130) deviennent des feuilles de papier, la polysémie du mot " feuille " fonctionnant aussi en allemand : " les feuilles ressemblaient à de vieux documents qui sentaient mauvais et avaient déjà commencé à se décomposer ${ }^{21}$ ". De même, l'évocation d'un paysage apocalyptique de déchets ("Unrat", 131) pourrait être guidée par le célèbre rapprochement opéré par Lacan entre les signifiants "letter " et "litter " (" ordure " en anglais), qu'il emprunte au cercle de James Joyce ${ }^{22}$. Flottant entre une logique associative descriptive et un jeu avec les signifiants, la matière oscille entre la réalité sensible du papier et celle des mots. Cette description magistrale ajoute aux effets narratifs de la lettre des effets poétiques mettant l'accent sur la langue dans sa matérialité. On serait proche alors des critères de la fonction poétique du langage développés par Roman Jakobson ${ }^{23}$. Pour autant, la fonction référentielle n'est pas exclue de ce passage qui, comme le texte-objet, permet une lecture à plusieurs niveaux. En effet, l'extrait peut se lire comme une description assez précise de la pollution en RDA, orchestrée par un régime prêt à dégrader son environnement et à compromettre la santé de sa population en faisant commerce des ordures, c'est-à-dire en acceptant de 1975 à 1989 de stocker les déchets de l'Ouest contre des devises ${ }^{24}$. Ainsi matière de la langue et matière des déchets, mais aussi matière de l'histoire allemande, se superposent-elles dans ce passage. Le recours à la matière permet ici de faire coïncider poésie et référence, en faisant pour ainsi dire l'économie du récit. Ce passage du texte objet en circulation au texte comme matière inaugure un modèle développé dans les récits ultérieurs de Hilbig. À partir des années

21. « Die Blätter glichen alten, übelriechenden Schriftstücken, die schon in Fäulnis übergegangen waren " (130).

22. Lacan, "La lettre volée ", p. 25-26.

23. Roman Jakobson, " Linguistique et poétique ", traduit par Nicolas Ruwet, in Jakobson, Essais de linguistique générale, Paris, Editions de Minuit, 1963, p. 209-248. Cf. Gérard Genette, Fiction et diction, Paris, Seuil, 2004 [1991], p. 103.

24. Stefan Wolle, "Müllimporte", in Wolle, Die heile Welt der Diktatur. Alltag und Herrschaft in der DDR 1971-1989, Bonn, Bundeszentrale für Politische Bildung, 1999, p. 207. 
1990, ces derniers se placent sous l'égide de la matière pour évoquer l'histoire allemande. Leur intrigue pourrait se résumer à la transformation de la matière ${ }^{25}$.

\section{Matière et récit dans Die Kunde von den Bäumen}

\section{De l'art de raconter à la crise du récit}

Die Kunde von den Bäumen (1994) est un livre sur la matière qui, dans le même temps, problématise explicitement la question du récit. La citation tirée de l'essai "Der Erzähler " de Walter Benjamin ${ }^{26}$ et située en exergue, place ainsi d'emblée le texte dans la perspective de l'art de raconter :

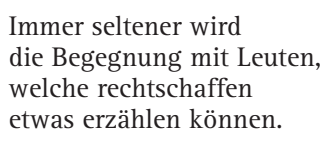

Cependant, la disposition typographique de l'épigraphe sème le trouble dans les catégories génériques. En effet, par le retour à la ligne, Hilbig semble choisir la forme d'un poème pour citer les propos de Benjamin sur le genre narratif. Die Kunde von den Bäumen inciterait à découpler la question de l'art de raconter de celle du genre littéraire à proprement parler. On peut y voir en germe le projet du recueil de poèmes Bilder vom Erzählen (2001) alliant récit et forme poétique. Le terme "Kunde " dans le titre du récit de Hilbig est un emprunt au même essai de Benjamin qui emploie ce mot désuet dans l'expression "die Kunde von der Ferne " et "die Kunde aus der Vergangenheit ${ }^{27}$ ". Signifiant " la nouvelle ", " le savoir qui vient de loin " ou " qui vient du passé ", ces tournures désignent l'expérience (au sens de "Erfahrung ") que ces formes archaïques de l'art du récit, incarnées respectivement par le marin et le paysan, parviennent à transmettre. Car pour Benjamin, l'art de raconter est un savoir-faire sur le point de disparaître, c'est d'ailleurs ce que dit la phrase qui précède la citation en exergue : "Elle [l'expérience] nous dit que l'art de raconter touche à sa fin ${ }^{28}$." Die Kunde von den Bäumen illustrerait la thèse benjaminienne liant la Première Guerre mondiale et l'épanouissement du capitalisme à la fin du récit oral traditionnel qui permettait la transmission de l'expérience humaine. Le texte de Hilbig se lirait comme son adaptation à la période du mur de Berlin.

C'est en effet sur une crise du récit que s'ouvre le texte. Le narrateur se trouve pris dans la tenaille d'une double impossibilité : l'impossibilité d'écrire

25. Citons Wolfgang Hilbig, Alte Abdeckerei, Frankfurt a. M., Fischer, 1991, " Die Arbeit an den Öfen " [1992], in Hilbig, Die Arbeit an den Öfen, Berlin, Friedenauer Presse, 1994, "Grünes Grünes Grab " [1992], in Hilbig, Grünes grünes Grab, Frankfurt a. M., Fischer, 1993.

26. Wolfgang Hilbig, Die Kunde von den Bäumen, Frankfurt a. M., Fischer, 1994. Walter Benjamin, " Der Erzähler. Betrachtungen zum Werk Nicolai Lesskows ", in Benjamin, Gesammelte Schriften II.2, Frankfurt a. M., Suhrkamp Verlag, 1977, p. 438-465, ici p. 439.

27. Ibid., p. 440.

28. "Sie [die Erfahrung] sagt uns, dass es mit der Kunst des Erzählens zu Ende geht », ibid., p. 439. 
des récits et l'impossibilité de ne pas écrire, c'est-à-dire l'injonction à raconter ("Erzähle!... erzähle! ", 9). Les premières pages du texte développent ainsi ce qui s'apparente aux topoï ou clichés de l'écriture du récit dans la modernité littéraire. C'est notamment la tendance à remplacer l'écriture du récit par le récit de son impossibilité qui les caractérise (8). Le texte de Hilbig alterne descriptions distanciées de cette tendance à la méta-littérature (11) et recours aux modèles conditionnant la survie à la faculté de raconter, tels Schéhérazade parvenant à ajourner son exécution en tenant le sultan captif de ses histoires $(11,12)$ ou encore le dieu de l'Apocalypse laissant à Jean le temps de terminer son livre avant de donner à ses armées l'ordre de fondre sur la terre (11). Dans le cas du narrateur, ce sont les histoires qui sont menacées d'oubli (12).

Cette crise du récit semble ainsi relever plus généralement d'une crise de l'écriture, d'un blocage. Le narrateur n'a écrit qu'une seule phrase, " L'allée aux cerises a disparu ${ }^{29}$ ", et se trouve incapable d'aller plus loin. Malgré ses dénégations, Die Kunde von den Bäumen est bien un texte métalittéraire qui expose et commente les difficultés qu'il a à s'écrire (10). Ce faisant, des éléments constitutifs du récit, comme la mise en scène de personnages (77) ou la question du temps dans le récit (40), font l'objet de commentaires explicites.

\section{De la matière du récit à la matière de l'histoire}

C'est avec la question de l'intrigue et du thème ou du sujet, c'est-à-dire de la matière du récit, que se nouent matérialité et narration dans Die Kunde von den Bäumen. La question encadre le livre et dessine un cheminement à travers la polysémie des mots. Ainsi, l'expression " intrigue de remplacement pour une matière manquante ${ }^{30}$ " fait correspondre " -handlung " et "Stoff " au début du livre. L'absence de matière, au sens d'intrigue, est à l'origine du blocage de l'écriture. À la fin du livre, le mot " matière " ("Stoff ") cède la place au mot "Vorwurf " (" sujet "), à l'expression " eine Idee zum Erzählen " (106) (" une idée à raconter "), et enfin au mot " Anlass " (106) (" prétexte ", " occasion "), qui peuvent se lire comme des variantes de l'idée d'intrigue, d'objet du récit. Le narrateur ne parvient pas à continuer son récit parce qu'il ne sait pas quoi écrire à propos des cerisiers. La solution réside dans le renversement du rapport sujet-objet : peu à peu, les cerisiers deviennent sujets du récit, et non plus objets, ce sont eux-mêmes qui racontent. Toute l'intrigue du récit de Hilbig, Die Kunde von den Bäumen, réside dans la transformation de la signification du groupe prépositionnel introduit par " von " : d'un sens " objet " à un sens " sujet ${ }^{31}$ ". Dans le même temps, le texte construit peu à peu l'étendue de la signification de la matière au sens de "Stoff". La matière manquante pour son récit est

\footnotetext{
29. "Die Kirschallee ist verschwunden " (30).

30. "Ersatzhandlung für den fehlenden Stoff " (8).

31. Cf. à ce sujet Graham Jackman, "The quest for a new language : Wolfgang Hilbig’s "Die Kunde von den Bäumen" ", in Graham Jackman, Ian F. Rol (dir.), Finding a voice. Problems of Language in East German Society and Culture, Amsterdam/ Atlanta, Rodopi, 2000, p. 247-271.
} 
transposée par le narrateur dans la métaphore du sol ("Boden "), ou plutôt de son absence, se faisant image du sol qui se dérobe ("Bodenlosigkeit »). Le glissement sémantique se poursuit, le sol apparaît constitué de matière usagée ("verbrauchte Materie ", 12), métonymie de l'histoire refoulée du pays. Archive d'un passé qu'on oublie, la matière s'incarne dans un lieu, une décharge en marge de la ville dont l'élément prédominant est la cendre. La lecture de l'histoire du point de vue de sa matière suscite une réinterprétation littérale du mot "Geschichte ", qui semble motiver à nouveaux frais (mais au prix d'une fausse étymologie) le mot lui-même en un accès de cratylisme ${ }^{32}$. L'histoire ("Geschichte ") apparaît alors comme le résultat d'une sédimentation, le fruit d'une superposition de couches de vivant ("Schichten " 13) désormais pétrifié.

L'histoire est ainsi matérialisée et spatialisée. Le récit enchâsse à l'intérieur du double sens de " matière " (objet d'un texte et substance réelle) une concordance similaire entre un terme de narratologie, " histoire " renvoyant au contenu du récit, et un terme connotant le réel, la chronologie des événements du passé. L'enjeu de Die Kunde von den Bäumen est le passage de la matière morte à une matière vive; la transformation d'un présent mort (" tote Gegenwart ", 13), " historique " (" nur noch Geschichte ", "leblos für immer ", 13), en une mémoire vivante qui nous " parle " - littéralement - encore aujourd'hui; la transmutation de la matière au sens de rebut (" Müll ") en la matière au sens de substance ou d'essence (" Substanz ", "Wesen "). Hilbig fait jouer le spectre sémantique de la matière dans son extension maximale. La décharge recouverte de cendre abrite des objets sans valeur et invendables (" unverkäufliche Dinge ", "Materialien ", 37), destinés au recyclage, tels que papier, bouteilles et ferraille. Dépouillées de leur usage et de toute utilité, c'est-à-dire de l'inessentiel, ces choses peuvent être considérées pour elles-mêmes. Elles se mettent à parler de leur substance, de leur essence même (61). Il faudrait alors considérer le texte de Hilbig comme le récit d'une épiphanie, c'est-à-dire le récit de la manifestation d'une vérité cachée dans ce qui semblait inerte et sans valeur : "Ce n'est que dans la cendre dans laquelle les arbres se sont retransformés, que leur essence est devenue manifeste ${ }^{33}$. " Dès lors, le récit de Hilbig opèrerait la transmutation de la matière en essence, du rebut en vérité. Le narrateur qualifie ce renversement d'" expérience quasi mythique " "fast mythische Erfahrung ", 61). La qualité de récit du texte hilbigien, son intrigue, semble résider dans cette transmutation de la valeur de la matière sur le modèle de l'alchimie.

\section{Une poétique de la matière à la manière de Lord Chandos}

Le récit est traversé de nombreux échos aux écrits de Benjamin. À la question de l'art de raconter s'ajoutent les références à la mélancolie et au langage des

32. En réalité, le mot " Geschichte " vient de " Geschick ", destin, événement.

33. "In der Asche erst, in die sich die Bäume zurückverwandelt haben, ist ihr Wesen offenbar geworden " (105). 
choses $^{34}$. Michael Opitz a aussi reconnu dans les personnages des éboueurs ("Müllmänner ") qui collectent les objets abandonnés une possible réécriture du chiffonnier, allégorie du poète moderne, décrit par Benjamin dans " Das Paris des Second Empire bei Baudelaire ${ }^{35}$ ". Cependant, à cette lecture benjaminienne se superpose le prisme du texte poétologique intitulé "Ein Brief " de Hugo von Hofmannsthal. La superposition de ces deux hypotextes constitue le creuset où s'élabore la poétique de la matière chez Hilbig.

La lettre fictive que Lord Chandos écrit au philosophe anglais Francis Bacon le 22 août 1603 est l'expression d'une crise de l'écriture qui est en même temps crise du langage et crise de la vision. Elle est sous-tendue par l'utopie d'un accès direct aux choses qui ne passerait pas par la langue, mais où le sujet serait en contact immédiat avec la matière. Dès le départ, l'accent est mis sur l'opposition entre la langue et les choses, sur l'insuffisance de la langue, sur son incapacité à pénétrer l'intérieur des choses (" ins Innere der Dinge zu dringen »). Les symptômes sont exprimés par des formules désormais célèbres :

Es ist mir völlig die Fähigkeit abhanden gekommen, über irgend etwas zusammenhängend zu denken oder zu sprechen. [...] die abstrakten Worte, deren sich doch die Zunge naturgemäß bedienen muß, um irgendwelches Urtheil an den Tag zu geben, zerfielen mir im Munde wie modrige Pilze ${ }^{36}$.

Elles résonnent dans les premières pages de Die Kunde von den Bäumen, notamment dans l'expression "l'incapacité de penser de manière abstraite ${ }^{37}$ ". Le narrateur fait état d'une crise du langage - les mots n'expriment pas ce qu'il veut nommer (7) -, et il s'inscrit dans la lignée des écrivains qui en ont fait le diagnostic (8). Hofmannsthal, comme Hilbig, fait des choses réputées de peu de valeur, abandonnées ou en train de se déliter (" un arrosoir, une herse abandonnée dans un champ ", " un cimetière misérable, un estropié ") les lieux d'une possible révélation ${ }^{38}$. On retrouve la même image des arbres destinés à disparaître, un pommier tordu chez Hofmannsthal ${ }^{39}$. C'est cette matérialité liée à la présence des choses que les mots semblent incapables de saisir et de rendre. Le souhait exprimé par Lord Chandos de ne faire qu'un avec ces objets matériels (" Materialien "), de se fondre en eux (" in [sie] hinüberfließen ") rejoint les

34. Cf. Walter Benjamin, " Über die Sprache überhaupt und über die Sprache des Menschen ", in Benjamin, Gesammelte Schriften, t. II. 1, p. 140-157, et Dorothee Kimmich, Lebendige Dinge in der Moderne, Konstanz, Konstanz University Press, 2011, p. 59 et 61 (sur l'écoute de la langue des choses chez Benjamin), p. 76 (sur la langue des choses chez Hofmannsthal).

35. Walter Benjamin, "Das Paris des Second Empire bei Baudelaire ", in Benjamin, Gesammelte Schriften, t. I. 2, p. 511-604, ici p. 521-522. Cf. Michael Opitz, " "Auf den düsteren Feldern des Abfalls" Peripheres als Zentrum. Wolfgang Hilbigs Die Kunde von den Bäumen ", in Martin Hellström, Edgar Platen (dir.), Zwischen Globalisierungen und Regionalisierungen. Zur Darstellung von Zeitgeschichte in deutschsprachiger Gegenwartsliteratur (V), München, Iudicium Verlag, 2008, p. 145-156.

36. Hofmannsthal, Brief, p. 465.

37. " [...] die Unfähigkeit abstrakt zu denken " (8).

38. "Eine Gießkanne, eine auf dem Feld verlassene Egge ", " ein ärmlicher Kirchhof, ein Krüppel ", " alles dies kann das Gefäß meiner Offenbarung werden. ", Hofmannsthal, "Brief ", p. 467.

39. " ein verkrümmter Apfelbaum ", ibid., p. 469. 
descriptions de la transformation du narrateur de Die Kunde von den Bäumen en statue de cendres, qui marque son assimilation progressive avec les hommes de la décharge et leur paysage $(89,96)$. Si la crise qui s'est emparée de Chandos ne lui laisse d'autre choix que d'abandonner la littérature et l'écriture des livres en général, c'est paradoxalement parce qu'il a trouvé une manière de penser en contact direct avec les choses et qui se passe des mots, " [une] pensée dans une matière qui est plus immédiate, plus fluide, plus incandescente que les paroles ${ }^{40}$ ". Pour le narrateur du texte hilbigien, en revanche, il s'agit toujours de " trouver une langue qui ne serait pas pré-formulée par l'État et ses administrateurs de la parole ${ }^{41}$ ". La dimension politique et historique est inhérente au récit de Hilbig, alors qu'elle n'est pas détectable dans la lettre d'Hofmannsthal. Cependant, il est possible de lire dans les dernières lignes de "Ein Brief ", l'un des cheminements centraux du texte de Hilbig : le passage du vertige dû à l'abîme sous les pieds (" das Bodenlose " chez Hofmannsthal, " die Bodenlosigkeit " (8) chez Hilbig) à la paix qu'offre le langage des arbres : "l'adoucissement de la colère " ("Glattwerden ihrer Wut ") chez Hilbig, "le giron [...] le plus profond de la paix " ("der tiefste[...] Schoß des Friedens ${ }^{42}$ ") pour Hofmannsthal. Et surtout, la même sortie de la crise - percevoir la langue des choses muettes chez Hofmannsthal ${ }^{43}$, celle des cerisiers chez Hilbig - se tient à l'horizon des deux textes.

Cette poétique de la matière trouve son expression dans le passage de Die Kunde von den Bäumen imitant la langue des cerisiers, langue sans objet, pur rythme suivant l'oscillation des marées et des saisons, racontant l'alternance du jour et de la nuit (107). L'hypotexte d'Hofmannsthal s'inscrit ainsi dans ces procédés de l'intertextualité non marquée qui traversent les textes de Hilbig sous la forme du dialogue, lui permettant de formuler et d'historiser un questionnement poétologique ${ }^{44}$.

\section{Faire (ou ne pas faire) des histoires}

Cependant, cette parole d'arbres n'est pas la fin du texte mais plutôt ce qui libère la parole et les souvenirs refoulés du narrateur. Au début du texte, les cerisiers sont ceux d'une allée séparant le village W. de ses marges. Situé sur des gisements de lignite, le village doit être déplacé afin de permettre la transformation du territoire en mine à ciel ouvert (23). Après la disparition

40. " [ein] Denken in einem Material, das unmittelbarer, flüssiger, glühender ist als Worte ", ibid., p. 471.

41. " eine Sprache [zu] finden, die von derjenigen abwich, die ihnen vom Staat, von den Verwaltern der Sprache, vorformuliert worden war " (81-82).

42. Hofmannsthal, "Brief ", p. 471.

43. " eine Sprache, in welcher die stummen Dinge zuweilen zu mir sprechen ".

44. Sur l'intertextualité non marquée, en particulier chez Hilbig, cf. Bärbel Heising, "Briefe voller Zitate aus dem Vergessen "Intertextualität im Werk Wolfgang Hilbigs, Frankfurt a. M., Peter Lang, 1996, p. 28, p. 58. Sur la présence du texte de Hofmannsthal dans Die Kunde von den Bäumen cf. Michael Opitz, Wolfgang Hilbig. Eine Biographie, Frankfurt a. M., Fischer, 2017, p.107. Voir aussi Wolfgang Hilbig, "Lieber Lord Chandos. Aufruf zum Widerstand. Warum wir dem Zerfall trotzen müssen ", Frankfurter Allgemeine Zeitung 165, 19 juillet 2002, p. 37. 
du village, l'allée de cerisiers, devenue inutile, menace d'être engloutie par la décharge qui la borde. Les cerisiers se lisent comme l'emblème de la politique est-allemande d'exploitation et de destruction des paysages. Peu à peu cependant, ils apparaissent comme le signe de l'irruption des événements de l'histoire de RDA dans la vie du narrateur. En effet, ce dernier empruntait l'allée des cerisiers avec ses camarades apprentis ouvriers jusqu'à ce qu'ils passent à l'Ouest (21-22). Les arbres rappellent au narrateur la " honte " ("Scham ", 113, 114) de n'avoir pas osé suivre ses camarades, d'être resté dans un pays qui, quelques temps après, fermait ses frontières, la "lâcheté " ensuite ("Feigheit ", 113) d'avoir survécu, de n'avoir pas serré autour de son cou la corde attachée à l'une des branches de ces cerisiers (112). Dans le sentiment de la honte réside la vraie raison du blocage de l'écrivain. La langue des arbres, qui se manifeste dans le mouvement et le frottement de la cendre, déclenche la prise de conscience de la manière dont le narrateur a été affecté par la construction du Mur en août 1961 et de la nature de cet affect. En ce sens, le " message " ("Kunde ") délivré par les arbres réside dans leur effet libérateur, plutôt que dans un contenu particulier. C'est aussi l'effet ou l'action concrète plutôt que les mots qui l'emportent à la fin du récit de Hilbig, - en ceci encore celui-ci reste proche de la langue imaginée par Hofmannsthal. Car le narrateur, qui a pourtant retrouvé l'inspiration, ne pourra pas poursuivre l'écriture de son récit en raison de contraintes matérielles : le tas de bois placé devant la hutte du narrateur s'anime et l'une des branches de cerisiers coupées tire la fiche de la lampe de la prise électronique.

\footnotetext{
Da glaubte ich, geblendet von dem andauernden Geflacker, einen Baumast gesehen zu haben, der sein Gezweig durch eine Spalt der Tür hereinschob und nach dem Kabel griff : der Kontakt wurde unterbrochen, das Licht ging zum dritten... zum vierten Mal aus ! - Macht keine Geschichten, murmelte ich. Mit dem Fuß schüttelte ich den Stecker, bis ich wieder Licht hatte. Doch der Ast - ohne Zweifel ein Stück aus dem Brennholzhaufen, der vor der Tür lag und aus den Überresten der Kirschallee stammte -, der Zweig, der an seinem Ende geformt war wie eine Hand, kam näher gekrochen. (117)
}

Du point de vue de la technique narrative, il s'agit là encore d'une forme de métalepse, l'objet du récit, les arbres, situés au niveau intradiégétique, entrent de manière impromptue dans le niveau-cadre, le niveau extradiégétique où l'on écrit. La dimension fantastique de cette fin du récit, l'image presque grotesque de la branche en forme de main, semblent discordantes par rapport au ton poétique et au style élevé du reste du texte. Pourtant, il est possible de lire dans ce geste une mise en œuvre de la poétique de la matière, en écho avec certains passages mettant l'accent sur l'effet (" Auswirkung ") de l'écriture, la transformation des mots en réalité ("verwirklichen ").

Der Satz [...] ist ganz und gar ohne eine Auswirkung geblieben : Die Bäume der Kirschallee sind verschwunden! [...] [E]rst wenn ich alle Geschichten dazu erzählt hätte, wären die Kirschbäume wieder da. (43) [E]s seien allein noch die Müllmänner, die in dieser Zeit einen poetischen Gedanken zu verwirklichen vermochten. (61)

Dans ce sens, la fin du texte de Hilbig met en pratique cette pensée poétique ou mythique qui consiste à faire revenir à la vie les cerisiers grâce à l'écriture du récit. 
L'expression " Macht keine Geschichten " (171) ("Ne faites pas d'histoires "), située au centre du paragraphe dans lequel est décrite l'action des cerisiers soudain ressuscités, se lit comme un calembour. Les branches de cerisiers font des histoires, "dépassent les bornes ", en ne restant pas sagement à leur place d'objet du récit, d'une part, parce qu'elles " font " littéralement le récit qu'elles ont repris en main et, d'autre part, parce qu'elles ont une valeur historique. Elles ressuscitent l'histoire du paysage autour du village de W. et font revivre l'émotion suscitée par la construction du Mur en 1961 chez le sujet écrivant. L’idiome "Geschichten machen " condense tous les signifiés du mot " Geschichte " accumulés au cours du texte. Aux strates renvoyant à la narration, puis à une évolution politique, sociale et culturelle pétrifiée et refoulée s'ajoute à présent une dimension performative, c'est-à-dire mettant en avant l'idée de " faire " : l'idée d'une littérature qui agit sur le réel. La tournure négative donne sans doute aussi à lire la manière hilbigienne d'écrire des fictions sur le mode de la dénégation, c'est-à-dire en exprimant ses doutes quant à la possibilité du récit.

\title{
Quelques enjeux de la matérialité chez Hilbig
}

Die Kunde von den Bäumen est un texte qui interroge la possibilité de l'écriture littéraire de l'histoire mais aussi plus généralement celle de l'écriture poétique en temps de dictature.

Le blocage que traverse le narrateur écrivain est lié à son incapacité à décrire la violence de l'événement que représente l'érection du mur de Berlin, au moment où il la subit. Elle va de pair avec le nécessaire après-coup de l'écriture : " Il ne m'était pas possible de décrire la violence des événements quand elle était à son apogée $^{45}$ ! " Parvenir à " décrire " (" beschreiben ") la violence condense l'enjeu de la référence dans Die Kunde von den Bäumen :

\begin{abstract}
[...] Schlieren von Bäumen, Gliedmaßen von Bäumen : so hat sie die Asche längst beschrieben! [...] - Und die Bäume müssen längst schon beschrieben werden wie Asche. Es ist klar, sie können anders überhaupt nicht beschrieben werden : und damit bleiben sie unbeschreiblich. - Der Gedanke an die Kirschbäume ist so schmerzlich wie ein Riß, von einem Nagel über das Glas gezogen, diagonal über das Glas, das einen Schrei ausstößt : Beschrieben! Und die Wirklichkeit scheint durchgestrichen für alle Zeit. - (105)
\end{abstract}

Comme souvent chez Hilbig, le retournement se situe dans la mise en scène du mot lui-même (" beschrieben werden ", " unbeschreiblich ", "Beschrieben! ") et le déploiement de sa polysémie. La première occurrence du verbe ("Schlieren [...], Gliedmaßen [...] beschreiben ") semble renvoyer à la troisième acception du verbe " beschreiben " dans le dictionnaire Duden, l'accomplissement du mouvement d'une courbe. Puis le paragraphe s'attache au paradoxe de la description de la destruction : comment décrire ce qui a disparu, ce qui n'est plus mais a subi un processus particulier? (" et ainsi ils demeurent indescriptibles "/ " und

45. " Es war mir nicht möglich, die Gewalt der Ereignisse zu beschreiben, wenn sie auf ihrem Höhepunkt war! " (114). 
damit bleiben sie unbeschreiblich »). Enfin, le retour au premier sens du verbe " beschreiben ", induit par la forme transitive du verbe et son préfixe be-, permet de résoudre la première aporie pour aboutir à un nouveau paradoxe : décrire signifie recouvrir d'écriture. La cendre décrit les arbres disparus parce qu'elle les recouvre. Mais cette forme de description-recouvrement semble de nouveau problématique car elle altère l'objet de la description, c'est-à-dire le réel : « Et la réalité semble rayée pour toujours ${ }^{46}$ ". Cependant, comme cette altération débouche sur la manifestation de l'essence des arbres, le récit semble plébisciter ce genre de description qui ne redouble, ni n'imite, ni ne représente le réel, mais le raye, l'impactant directement, à la manière d'un performatif.

Ainsi, les mises en scène de la matière explorent de possibles réponses à un questionnement poétologique à propos de la référence qui traverse la prose de Hilbig depuis ses débuts. Le performatif mettrait en œuvre ce que, dans l'un de ses essais, l'auteur a pu appeler une "idée mythique " (" eine mythische Idee "), qu'il définit en 1982 comme la création des choses par les mots : "Dans la seule idée que ce sont d'abord les mots qui créent les choses - idée mythique -, la pensée que les mots ne sont 'pas' le simple medium des pouvoirs prend une forme empirique ${ }^{47}$. " Dans ce pouvoir qu'ont les mots de passer les frontières du texte et de faire advenir littéralement les choses dans la vie, comme le texte du narrateur hilbigien parvenant à transformer un ouvrier en écrivain, un écrivain en criminel, ou à animer les branches de cerisiers morts, il faut voir une forme de résistance des mots à la manipulation à laquelle ils sont soumis aux heures sombres de l'histoire.

Die Kunde von den Bäumen se lit en effet comme une possible réplique au poème de Bertolt Brecht "An die Nachgeborenen ", publié en 1939 dans les Svendborger Gedichte ${ }^{48}$, où on trouve les vers suivants :

Was sind das für Zeiten, wo

Ein Gespräch über Bäume fast ein Verbrechen ist

Weil es ein Schweigen über so viele Untaten einschließt !

Brecht y défend la posture de l'écrivain en colère, engagé dans les conflits de son temps et de ses contemporains. Il proclame l'impossibilité de la position du sage et d'un traitement apaisé des thèmes traditionnels de la poésie (l'amour, la nature), qui reviendrait à se faire complice des crimes de son temps. À l'engagement dans les combats, Hilbig oppose une implication au plus près de la destruction qui redonne la parole aux choses disparues. Au sentiment de la colère chez Brecht répond chez Hilbig celui de la honte auquel succède l'apaisement. Le ton

46. " Und die Wirklichkeit scheint durchgestrichen für alle Zeit », p. 105. " durchgestrichen " pourrait signifier aussi " passer au crible ». Hilbig joue sans doute sur la polysémie du verbe. Cependant, la phrase qui précède évoque la fissure provoquée par un clou sur un verre, qui fait davantage songer à une rayure qu'à un crible.

47. "Allein in der Idee, dass erst die Wörter die Dinge erschaffen - eine mythische Idee -, gewinnt der Gedanke daran, dass die Wörter nicht bloßes Medium der Gewalten sind, eine empirische Form. " Wolfgang Hilbig, " Der Mythos ist irdisch. Für Franz Fühmann zum 60. Geburtstag ", in Hilbig, paradiesen, p. 202-211, ici p. 209.

48. Bertolt Brecht, Die Gedichte, éd. par Jan Knopf, Frankfurt a. M., Suhrkamp, 2007, p. 355-357. 
poétique de la prose de Hilbig réplique au ton prosaïque du poème de Brecht. Écrire une littérature de l'implication qui fait parler les choses serait la forme de l'engagement littéraire selon Hilbig. Dans la dernière de ses conférences de Francfort en 1995, il écrit à propos de la littérature : " elle voulait faire parler les choses - en provoquant ou en protestant -, elle voulait quoi qu'il en soit prendre part au malheur de ce monde ${ }^{49} "$.

Les essais de 1982 et de 1995 ne disent certes pas exactement la même chose et le recueil Der Brief et le texte Die Kunde von den Bäumen ne se ressemblent pas. Il n'en reste pas moins que le truchement de la matière permet à Hilbig de répondre à un même problème, celui de la référence, en mettant en œuvre ce que l'on pourrait appeler des poétiques de la participation qui cherchent à faire trembler la frontière entre le sujet écrivant et son objet, entre la littérature et le monde, et parfois même à l'abolir. Ces textes de Hilbig ont donc en commun d'être mus par un questionnement à la fois théorique (la référence) et existentiel (le rapport de la littérature à la vie). Leur écriture s'apparente à un processus de recherche. Ils font référence aux textes théoriques et poétologiques de Benjamin et Hofmannsthal. En cela, ils ressemblent à des essais. L'insistance sur la matière du texte leur confère une dimension poétique. Mais les réponses qu'ils mettent en œuvre (le performatif, la participation) nécessitent souvent les dispositifs de la fiction que sont la délégation de la parole à un personnage, ne serait-ce qu'un narrateur soliloquant, et l'esquisse de deux niveaux distincts (intra- et extradiégétique), ce en quoi ils se rattachent au récit.

49. " [S]ie wollte die Dinge zur Sprache bringen - provozierend oder protestierend -, sie wollte sich immerhin am Unglück dieser Welt beteiligen. " Wolfgang Hilbig, Abriss der Kritik. Frankfurter Poetikvorlesungen, Frankfurt a. M., Fischer, 1995, p. 98. 
